\title{
Sistema electrónico de recuperación de aguas grises por fotocatálisis solar
}

\section{para riego de cultivos.}

\author{
Electronic gray water recovery system by solar photocatalysis for crop irrigation.
}

Sistema eletrônico de recuperação de água cinza por fotocatálise solar para irrigação de plantações.

\section{ARTÍCULO GENERAL}

Hipólito carbajal morán hipolito.carbajal@unh.edu.pe

Universidad nacional de huancavelica. Perú

\author{
Javier francisco márquez \\ camarena \\ javier.marquez@unh.edu.pe \\ Universidad nacional de \\ huancavelica. Perú \\ Marco aurelio rosario \\ villarreal \\ marco.rosario@unh.edu.pe \\ Universidad nacional de \\ huancavelica. Perú
}

Carlos abel galván maldonado carlos.galvan@unh.edu.pe Universidad nacional de huancavelica. Perú

Recibido 12 de Setiembre 2021 | Arbitrado y aceptado 12 de Setiembre 2021 | Publicado en 12 de Octubre 2021

\section{RESUMEN}

Este artículo presenta el diseño y la implementación de Objetivos: un sistema electrónico de recuperación de aguas grises por fotocatálisis solar para riego de cultivos. Material y métodos: como parte de los materiales se empleó el PLC Simatic S7 1500, módulo analógico de 8 canales interconectados a dispositivos de medición online: sensor de radiación UV, transductor de $\mathrm{pH}$, transductor de conductividad eléctrica (EC), transductor de oxígeno disuelto (DO) y transductor de cloro libre (FCL). Se empleó el método inductivo-deductivo y ensayo error, siendo la unidad de análisis la calidad de aguas grises tratadas. Resultados: la posición geoespacial de depurador fue determinado adecuadamente por un sistema de posicionamiento global siendo la inclinación considerada $12^{\circ}$ al norte, el índice de radiación UV solar llega hasta 14, el periodo de exposición es programable desde una interface HMI de acuerdo a las necesidades, logrando como resultado que el agua gris tenga la mayor exposición a la radiación solar durante el día para su recuperación. Conclusiones: el sistema electrónico expone al agua gris a la máxima radiación solar de acuerdo a los requerimientos, lo que permitirá recuperar el agua mejorando los parámetros de acuerdo al estándar de calidad ambiental para agua (ECA-AGUA).

Palabras claves

PLC, sensores, calidad de agua, radiación UV solar.

\section{ABSTRACT}

This article presents the design and implementation of Objectives: an electronic system for the recovery of gray water by solar photocatalysis for irrigation of crops. Material and methods: as part of the materials, the Simatic S7 1500 PLC was used, an 8-channel analog module interconnected to online measurement devices: UV radiation sensor, $\mathrm{pH}$ transducer, electrical conductivity (EC) transducer, dissolved oxygen transducer (DO) and free chlorine transducer (FCL). The inductive-deductive method and trial error were used, the unit of analysis being the quality of treated gray water. Results: the geospatial position of the scrubber was adequately determined by a global positioning system, the inclination being considered $12^{\circ}$ to the north, the solar UV radiation index reaches 14 , the exposure period is programmable from an $\mathrm{HMI}$ interface according to the needs, achieving as a result that gray water has the greatest exposure to solar radiation during the day for its recovery. Conclusions: the electronic system exposes the gray water to the maximum solar radiation according to the requirements, which will allow to recover the water by improving the parameters according to the environmental quality standard for water (ECA-AGUA).

Keywords

PLC, sensors, water quality, solar UV radiation.

\section{RESUMO}

Este artigo apresenta a concepção e implementação dos Objetivos: um sistema eletrônico de recuperação de água cinza por fotocatálise solar para irrigação de lavouras. Material e métodos: como parte dos materiais, foi utilizado o Simatic S7 1500 PLC, um módulo analógico de 8 canais interligado a dispositivos de medição online: sensor de radiação UV, transdutor de $\mathrm{pH}$, transdutor de condutividade elétrica (EC), transdutor de oxigênio dissolvido (DO) e transdutor de cloro livre (FCL). Foram utilizados o método indutivo-dedutivo e o erro de tentativa, sendo a unidade de análise a qualidade da água cinza tratada. Resultados: a posição geoespacial do lavador foi determinada adequadamente por um sistema de posicionamento global, sendo a inclinação considerada $12^{\circ}$ para o norte, o índice de radiação UV solar chega a 14 , o período de exposição é programável a partir de uma interface HMI de acordo com as necessidades, conseguindo como resultado que a água cinza tem a maior exposição à radiação solar durante o dia para sua recuperação. Conclusões: o sistema eletrônico expõe a água cinza à radiação solar máxima de acordo com os requisitos, o que permitirá a recuperação da água melhorando os parâmetros de acordo com a norma de qualidade ambiental da água (ECA-AGUA).

Palavras chaves

PLC, sensores, qualidade da água, radiação UV solar. 


\section{Introducción}

El agua es un recurso indispensable para las actividades humanas, para el desarrollo económico y el bienestar social (Mehta, 2014). En promedio se necesitan tres mil litros de agua por persona para generar los productos necesarios para la alimentación diaria (FAO, 2017). Aunque la irrigación para fines agrícolas representa apenas $10 \%$ del agua usada, esta es la actividad de mayor consumo de agua dulce del planeta (Silva et al., 2008).

Las aguas grises son una importante fuente adicional para satisfacer la demanda del recurso para riego de cultivos (Franco, 2007), a causa de la falta de agua potable para cubrir los requerimientos de las poblaciones, los bajos costos, los beneficios para los suelos agrícolas y la disminución del impacto sobre el ambiente. Sin embargo, el predominio del uso de aguas contaminadas en el Perú, generan riesgos en la salud pública, en especial cuando se utilizan para riego de cultivos y para consumo directo (Larios et al., 2015).

A nivel mundial, encontramos el aprovechamiento de las aguas grises de las viviendas en diferentes actividades domésticas (Valencia et al., 2010). Es así como en varios países del mundo se han usado éstas en actividades que no requieren agua de calidad potable. Según investigaciones el reúso del agua conlleva a ahorros de entre 30 y $50 \%$ en el consumo de agua potable en las viviendas (EMRC, 2011).

Se realizaron diversos estudios que sirvieron de base a esta investigación como el desarrollado por A. Barwal y R. Chaudhary (2016) que implementan un reactor acoplado a un sistema parabólico diseñado para remover la carga orgánica y la desinfección microbiana de aguas contaminadas el efluente es sometido a una degradación orgánica, luego es sometido a la energía solar para la degradación bacteriana sometiendo a radiaciones en pleno Sol de 400 a $700 \mathrm{~W} / \mathrm{m}^{2}$ y en días nublados de 170 a $250 \mathrm{~W} / \mathrm{m}^{2}$ durante un periodo de tiempo de 5 a 6 horas con una eficiencia de $40 \%$ y $13 \%$ respectivamente.

Se entiende por depuración microbiana de aguas grises a los procesos físicos químicos y biológicos con la intervención final de la radiación solar, el que debe reducir a cierto nivel la contaminación microbiana del agua residual para que no exceda la 
Hipólito Carbajal Morán, Javier Francisco Márquez Camarena, Marco Aurelio Rosario Villarreal, Carlos Abel Galván
Maldonado

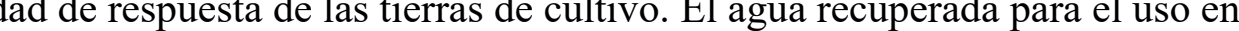
cultivos de plantas debe tener ciertas características físico-químicos(FAO, 2017).

La mayor preocupación para implementar la utilización de este tipo de aguas en el riego de cultivos, son los compuestos químicos y los microorganismos que podrían ser nocivos para la salud (Niño \& Martínez, 2014); por tal razón es necesario cumplir con los estándares de uso de agua. Para la correcta reutilización adecuándolas por medio de sistemas de tratamiento; siendo posible el uso en riego de cultivos y al interior de las viviendas, siempre y cuando se tomen las medidas de seguridad pertinentes.

Debido a esta necesidad de aprovechar las aguas grises se planteó el estudio teniendo como objetivo diseñar e implementar un sistema electrónico para la recuperación de estas aguas empleando la radiación solar y exponiendo por determinados periodos programados previamente.

\section{Materiales y métodos}

El estudio fue del tipo experimental de carácter cuantitativo, para evaluar las variables de estudio se aplicó el método deductivo - inductivo mediante el análisis y síntesis. Para el logro de los objetivos se trabajó con datos que caracterizan a las variables provenientes de las pruebas experimentales y los análisis físicos-químicos de las aguas grises.

Por su naturaleza experimental el trabajo de investigación corresponde al nivel explicativo (Cegarra Sánchez, 2012), orientado a obtener información cuantitativa de las variables en estudio. Se evaluó el grado de intervención de las variables para recuperar las aguas grises.

\section{Implementación del sistema electrónico}

Se diseñó e implemento el sistema electrónico compuesto por un PLC S7 1500 que es del tipo modular, con rendimiento medio y superior. Con rendimiento adecuado para aplicaciones industriales y de laboratorio (Peciña, 2018). Dependiendo de las necesidades el controlador puede ampliarse mediante módulos de entrada/salida, tanto para señales digitales como analógicas y módulos tecnológicos específicos y de comunicación(Peciña, 2018). 


\subsubsection{Sensores - transmisores empleados}

\subsubsection{Sensor - transmisor de $p H$}

El sensor de $\mathrm{pH}$ de la figura 1, es un elemento que detecta corriente generada por presencia de iones de hidrógeno en el agua. La medida está en el rango de 0-14. Se emplea en este trabajo el sensor de pH en conjunción con el transmisor, bajo la certificación de protección IP 68 para inmersión continua en agua (BOPLA, 2019), el rango de temperatura es de 0 a $60{ }^{\circ} \mathrm{C}$, la comunicación de las medidas es por señal analógica de $4 \mathrm{~mA}$ a $20 \mathrm{~mA}$.

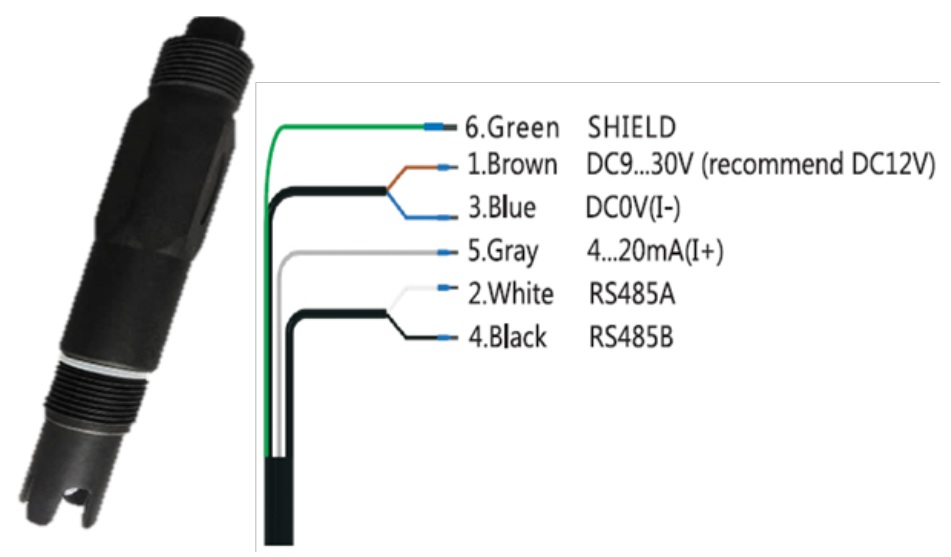

Figura 1. Sensor y transmisor de $\mathrm{pH}$

\subsubsection{Sensor - transmisor de conductividad eléctrica}

La conductividad eléctrica relaciona la concentración de iones provenientes de sales disueltas y materia orgánica en el agua. Las unidades en la que se mide están dadas en microsiemens por centímetro $\left(\frac{\sqrt{\mathrm{cm}})}{\mathrm{cm}}\right)($ Boyd, 2020) El sensor que mide esta conductividad tiene sonda electrónica que aplica voltaje en sus extremos para medir la resistencia del agua y convertir en conductividad .

\subsubsection{Sensor - transmisor de oxígeno disuelto}

El oxígeno disuelto (DO) representa la cantidad de oxígeno gaseoso disuelto $\left(\widehat{O_{2}}\right)$ en la solución (aguas grises) (Boyd, 2020). Las muestras se midieron online sus unidades están dados en miligramos por litro (mg/L) (REMOND, 2019). La cantidad de 
DO necesario en el agua para riego de cultivos es $\geq 4 \mathrm{mg} / \mathrm{L}$ (MINAM, 2017b). El $\mathrm{El}^{\text {Maldonado }}$ dispositivo empleado se ve en la figura 2.

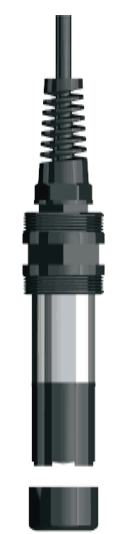

Figura 2. Sensor-transmisor de DO

\subsubsection{Sensor - transmisor de cloro libre}

El cloro libre tiene la capacidad de reaccionar con iones de amoniaco y con los compuestos orgánicos, formando el cloro combinado. La suma del cloro combinado con el cloro residual da el cloro total (Boyd, 2020). El cloro residual (FCL), tiene como unidad de medición el miligramo por litro por partes por millón (mg/L ppm). La cantidad de FCL necesario en el agua para riego de cultivos es FCL $\leq 3 \mathrm{mg} / \mathrm{L}$ (ppm) (FAO, 2017). El dispositivo usado se ve en la figura 3.

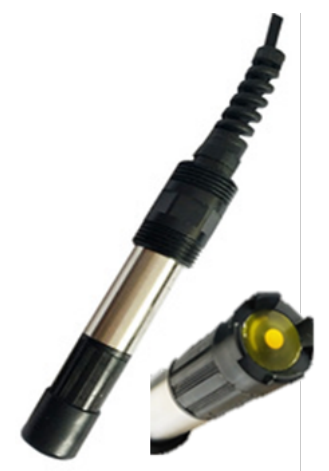

Figura 3. Sensor-transmisor de cloro residual 


\subsubsection{Sensor de radiación $U V$}

El sensor de radiación UV permite determinar la irradiancia sobre la superficie del fotorreactor con longitud de onda del espectro UV de $240 \mathrm{~nm}$ a $380 \mathrm{~nm}$ (Figura 4). Se emplea en la investigación el sensor UV 30A, que facilita determinar esta radiación de acuerdo al estándar de la Organización Mundial de la Salud (OMS).

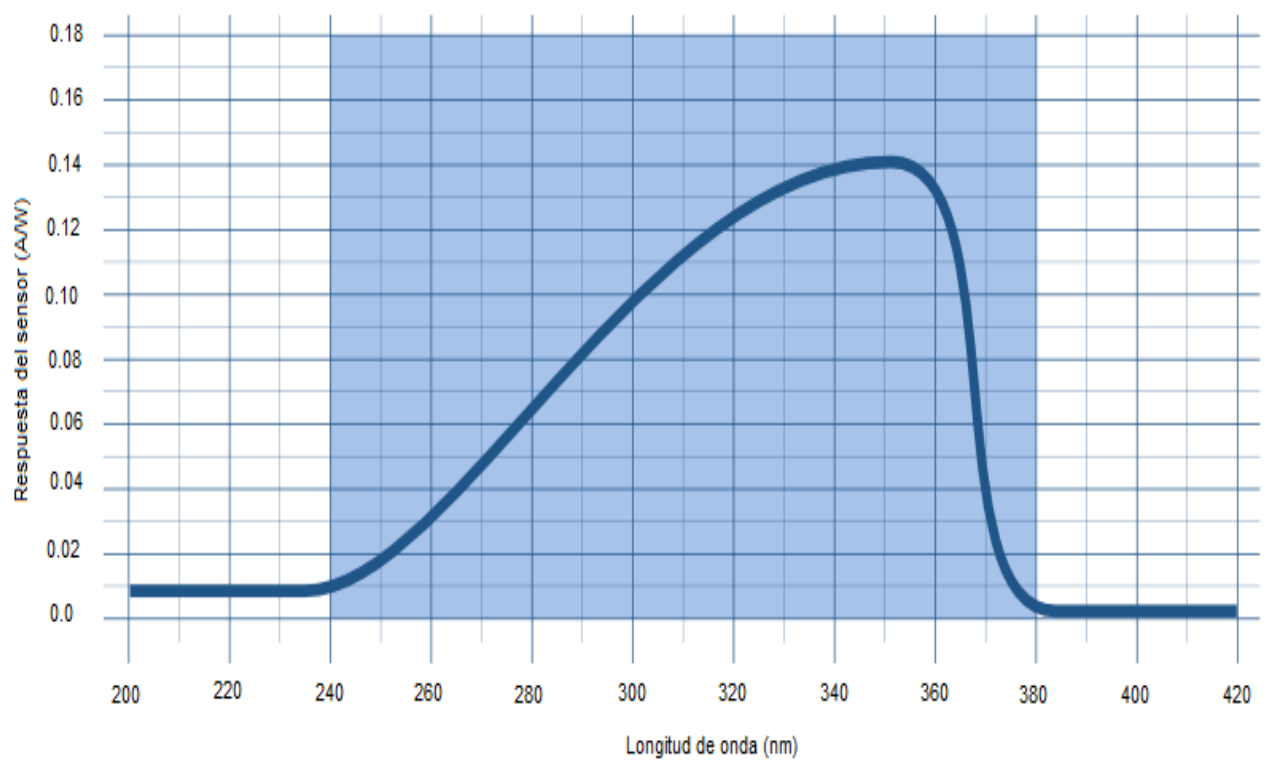

Nota: La respuesta del sensor abarca el espectro de la radiación UVA y UVB. Imagen obtenida de (Ventura, 2016).

Figura 4. Capacidad de respuesta del sensor de radiación UV 30A.

\subsubsection{Adquisición de datos}

El controlador lógico programable (PLC) SIMATIC S7 1500 es uno de los componentes importantes para la adquisición de datos, es del tipo modular, con rendimiento adecuado para aplicaciones industriales y de laboratorio, interactuando con módulos de entrada/salida (Figura 5), tanto para señales digitales como analógicas (Peciña, 2018). 


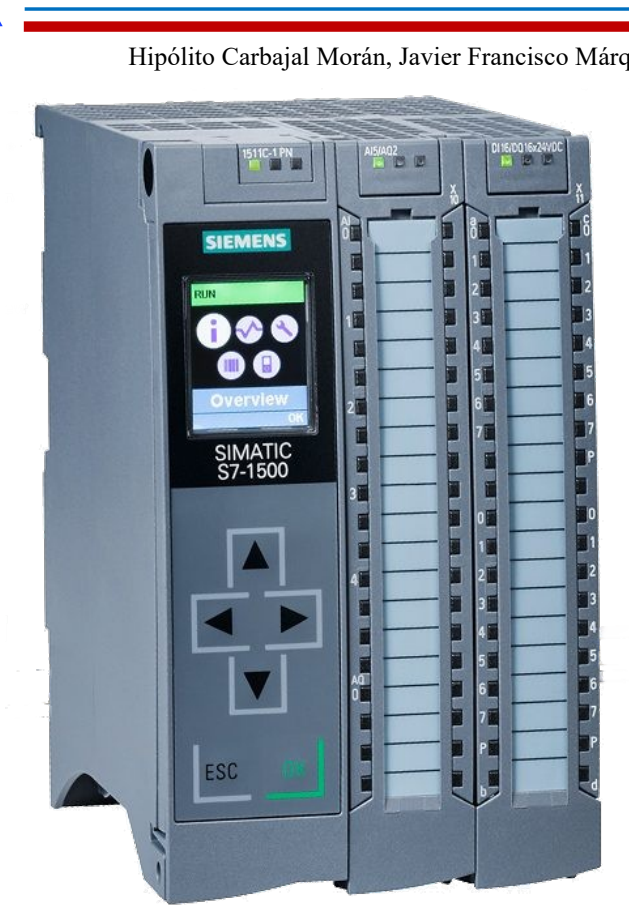

Nota: En la imagen el componente de la izquierda es la CPU del PLC SIMATIC S7 1500; el componente del centro es el módulo de entrada analógico y el componente de la derecha es el módulo de entrada/salida digital (Siemens, 2013)

Figura 5. PLC SIMATIC S7 1500 y módulos de entrada/salida

En la figura 6, los sensores - transmisores que miden pH, EC, DO y FCL se interconectan a 4 hilos al canal $0(\mathrm{CH} 0)$, canal $1(\mathrm{CH} 1)$, canal $2(\mathrm{CH} 2)$ y canal $3(\mathrm{CH} 3)$ respectivamente. Del mismo modo el sensor de radiación UV se conectó al canal 4 (CH4) configurado para señal de tensión (5V). 


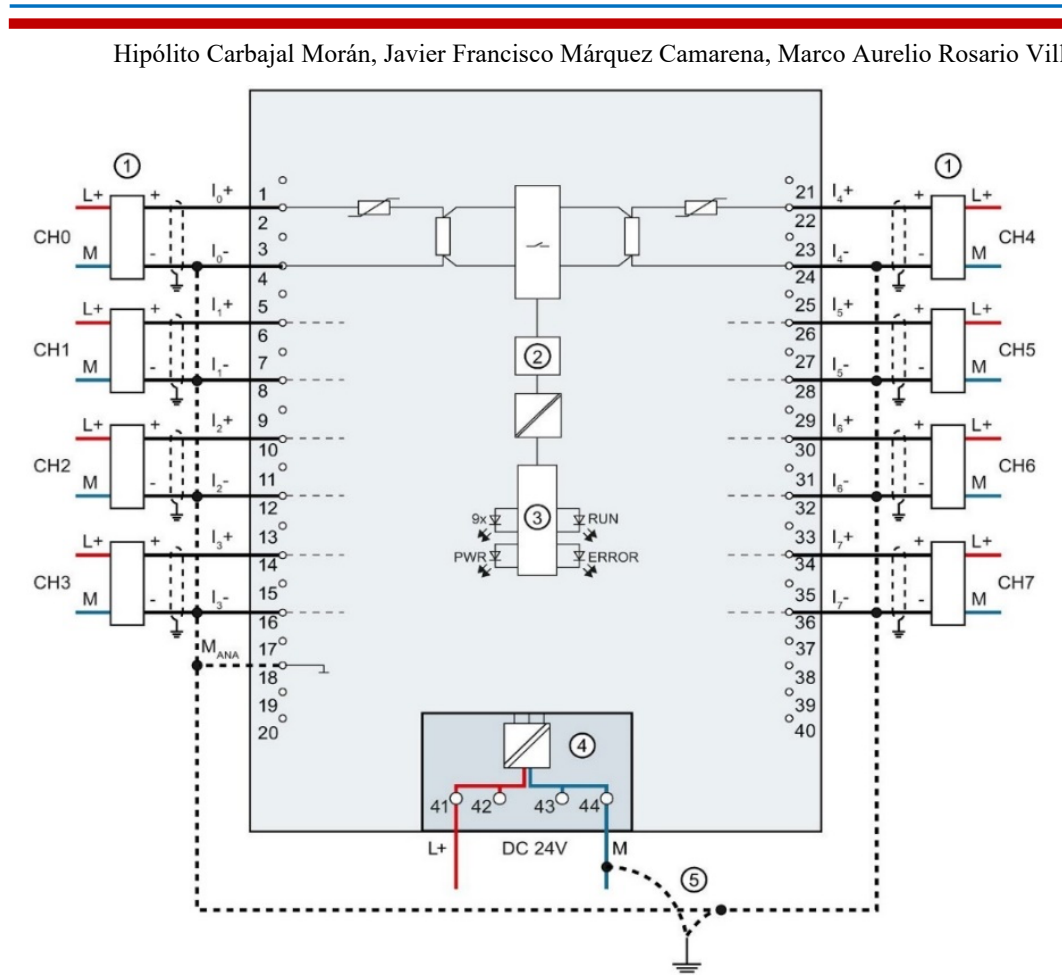

Figura 6. Configuración del módulo analógico para $S 7$ 1500, 4 hilos para medida de intensidad de 4 mA a 20 mA (Siemen, 2018).

Los datos se adquieren desde el bloque sensores de la figura 7, implementado en el TIA Portal (Siemens, 2017), cuyo programa fue desarrollado en Ladder, conteniendo una etapa de normalización y escalamiento de acuerdo al rango de medición de cada instrumento (sensor-transmisor) empleado

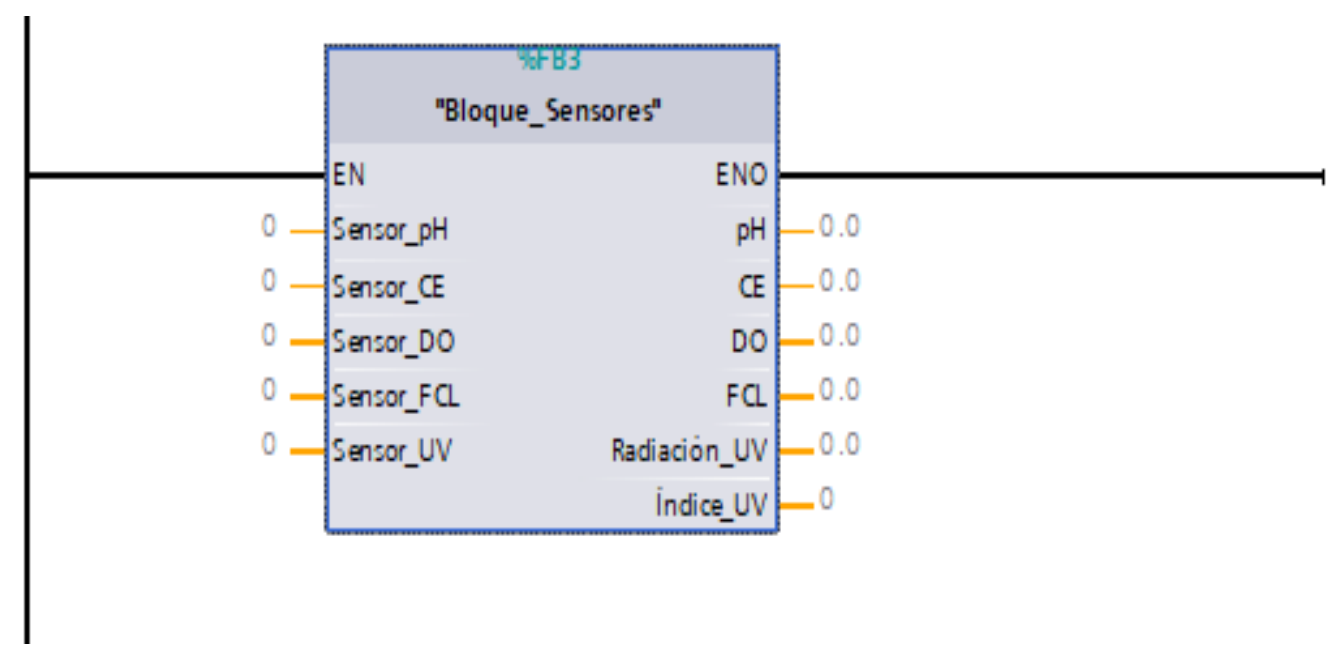

Figura 7. Bloque de sensores, implementado para PLC S7 1500 y módulo analógico. 


\subsubsection{Interface HMI}

Hipólito Carbajal Morán, Javier Francisco Márquez Camarena, Marco Aurelio Rosario Villarreal, Carlos Abel Galván

La programación del tiempo de exposición es llevado a cabo desde la interface HMI desarrollado en Wincc del TIA Portal (Siemens, 2017). Desde el tablero de control se activa el proceso para la exposición a la radiación solar de las aguas grises en tratamiento. El almacenamiento de los datos de los diferentes parámetros del agua son almacenadas en forma automática o manual cada 15 minutos en la memoria SMC de 4 MB.

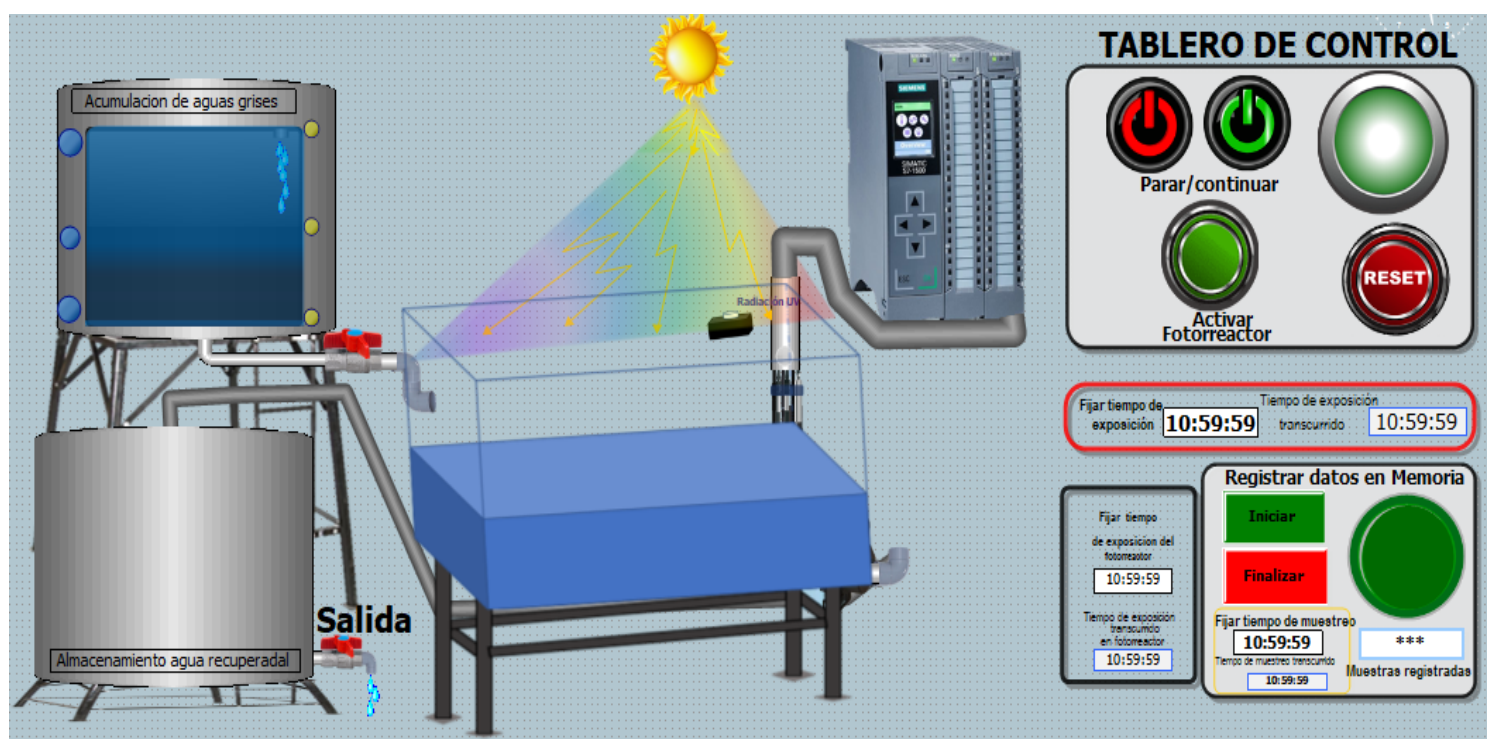

Figura 8. Interface HMI del tablero de control y fotocatálisis solar del sistema electrónico implementado.

\section{Población de estudio y muestra}

La población de estudio estuvo conformada por $1 \mathrm{~m}^{3}$ de volumen de agua gris obtenidos para este fin con la mezcla de detergente, jabón y blanqueadores.

Cada muestra fue de 10 litros de agua gris, se trabajó con 10 muestras. En cada muestra se determinaron los indicadores de: concentración de iones de hidrógeno $(\mathrm{pH})$, conductividad eléctrica (CE), oxígeno disuelto (DO), cloro libre (FCL). La toma de muestras se realizó únicamente en los puntos de monitoreo que fueron la entrada al sistema electrónico y salida del mismo, estos puntos fueron debidamente establecidos por los periodos de monitoreo desarrollados en Wincc. 
La variable experimental está constituida por los indicadores: irradiancia UV solar y el tiempo de exposición del agua a la radiación solar. Se implementa los parámetros de entrada y los parámetros de salida. La función astronómica, permite obtener una irradiación solar optimizada, lo que nos proporciona niveles altos de los indicadores de irradiancia UV solar (SENAMHI, 2019).

En la investigación se usan fotodiodos de tipo Schottky incorporado a una etapa amplificadora del módulo 30A, que comunica con señales analógicas el resultado de la medición de la radiación UV solar. El rango de longitudes de onda empleados en la investigación abarca de $240 \mathrm{~nm}$ a $380 \mathrm{~nm}$.

Se evaluó la variable experimental, agua gris tratada, correlacionando las variables empleando la estadística descriptiva, utilizando el software SPSS V26. Así también se utilizó las técnicas de validación y confiabilidad teórica dentro del paradigma cuantitativo.

\section{Resultados}

El fotocatalizador solar que es parte del sistema electrónico fue posicionado empleando un GPS NEO 6M, en la latitud $-12.391096^{\circ}$, longitud $-74.858124^{\circ}$ y a una altura de $3,285 \mathrm{msnm}$ con una tolerancia de $\pm 10 \mathrm{msnm}$.

A la entrada del proceso las aguas grises fueron caracterizados presentando un $\mathrm{pH}$ de 9.12, CE $2720 \mathrm{uS} / \mathrm{cm}$, DO $1.22 \mathrm{mg} / \mathrm{L}$, FCL $3.60 \mathrm{mg} / \mathrm{L}$ y una radiación en el fotocatalizador de $1,015.98 \mathrm{~mJ} / \mathrm{cm}^{2} \mathrm{~h}$ que marca el inicio del tiempo de exposición 11:00 am, estando programado el sistema electrónico para almacenar el valor de los parámetros en medición cada 15 minutos, siendo los resultados obtenidos lo que se muestra en la tabla 1. 
Tabla 1. Medición de parámetros de las aguas grises en tratamiento de

11:00 horas a 14:00 horas

\begin{tabular}{lllllll}
\hline $\begin{array}{l}\text { Tiempo de } \\
\text { exposición }\end{array}$ & $\begin{array}{l}\text { Duración } \\
\text { de } \\
\text { exposición } \\
\text { (min) }\end{array}$ & $\begin{array}{l}\text { Radiación } \\
\text { UV } \\
\mathrm{mJ} / \mathrm{cm} 2 \mathrm{~h}\end{array}$ & $\mathrm{pH}$ & $\begin{array}{l}\mathrm{EC} \\
(\mathrm{uS} / \mathrm{cm})\end{array}$ & $\begin{array}{l}\mathrm{DO} \\
(\mathrm{mg} / \mathrm{L})\end{array}$ & $\begin{array}{l}\text { FCL } \\
(\mathrm{mg} / \mathrm{L})\end{array}$ \\
\hline $11: 00$ & 0 & 1015.98 & 9.12 & 2720.00 & 1.22 & 3.60 \\
$11: 15$ & 15 & 1063.06 & 9.00 & 2715.20 & 1.22 & 3.50 \\
$11: 30$ & 30 & 1115.10 & 8.80 & 2700.20 & 1.30 & 3.40 \\
$11: 45$ & 45 & 1153.92 & 8.60 & 2680.00 & 1.50 & 3.20 \\
$12: 00$ & 60 & 1174.57 & 8.55 & 2650.00 & 1.54 & 3.00 \\
$12: 15$ & 75 & 1174.57 & 8.53 & 2600.70 & 1.57 & 2.80 \\
$12: 30$ & 90 & 1174.57 & 8.52 & 2657.00 & 1.54 & 2.50 \\
$12: 45$ & 105 & 1115.10 & 8.51 & 2655.00 & 1.58 & 2.30 \\
$13: 00$ & 120 & 1032.50 & 8.50 & 2650.90 & 1.58 & 2.00 \\
$13: 15$ & 135 & 991.20 & 8.48 & 2620.00 & 1.80 & 2.30 \\
$13: 30$ & 150 & 929.25 & 8.45 & 2618.00 & 2.00 & 2.20 \\
$13: 45$ & 165 & 867.30 & 8.30 & 2590.20 & 2.30 & 1.80 \\
$14: 00$ & 180 & 826.00 & 8.10 & 2592.00 & 2.40 & 1.20 \\
\hline
\end{tabular}

El agua para riego de cultivos no debe exceder ciertos niveles máximos permisibles (Akiça, 2004; MINAM, 2017b): pH de 6.5 a 8.5, $\mathrm{CE}<2500, \mathrm{DO} \geq 4$ y FCL $<$ 1. Por que se hace el análisis de regresión lineal a cada parámetro de la tabla 1, para ver si existe la variación de los valores en función al tiempo de exposición y la irradiancia UV solar.

Del análisis de regresión lineal para el parámetro $\mathrm{pH}$ se obtiene los estadísticos de bondad de ajuste (tabla 2), el mismo que da un índice de determinación de $\mathrm{R}^{2}=0.835$, que indica que el $\mathrm{pH}$ es dependiente del Tiempo de exposición en minutos en un $83.5 \%$ y se debe a otros factores en un $6.5 \%$.

Tabla 2. Estadísticos de bondad de ajuste para $\mathrm{pH}$

\begin{tabular}{ll}
\hline Observaciones & 13.000 \\
Suma de los pesos & 13.000 \\
GL & 11.000 \\
$\mathrm{R}^{2}$ & 0.835 \\
$\mathrm{R}^{2}$ ajustado & 0.820 \\
\hline
\end{tabular}


Hipólito Carbajal Morán, Javier Francisco Márquez Camarena, Marco Aurelio Rosario Villarreal, Carlos Abel Galván

La figura 9 muestra la regresión lineal del pH con una línea de tendencia con pendiente negativa (-0.0042), lo que indica que aun podrá disminuir el $\mathrm{pH}$ a medida que se incremente el tiempo de exposición.

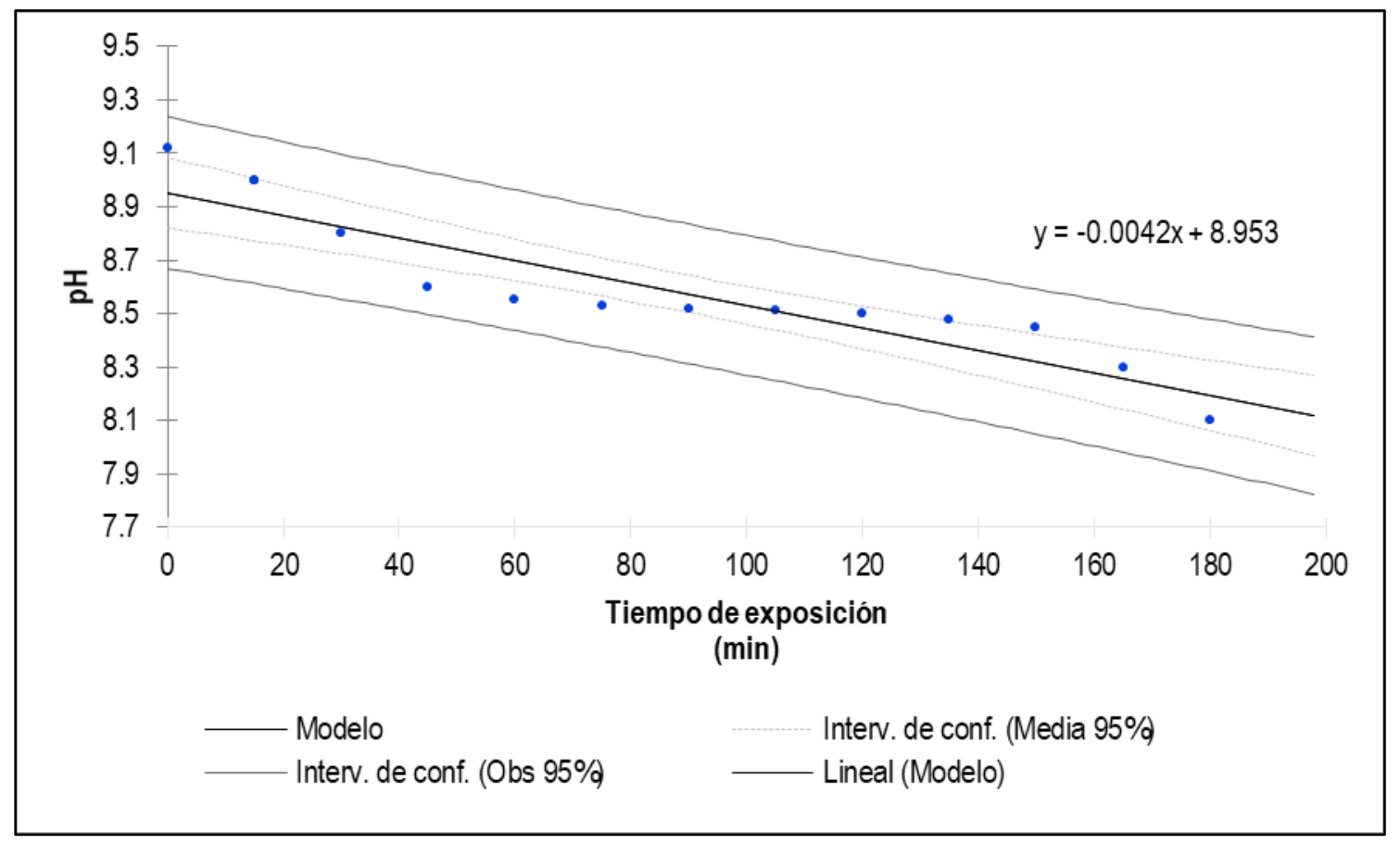

Figura 9. Regresión lineal del pH por Tiempo de exposición

En la tabla 3 se presenta el análisis de varianza para el $\mathrm{pH}$; dado el valor $\mathrm{p}(<$ 0.0001) asociado al estadístico F calculado (55.639) en la tabla ANOVA, y dado el nivel de significación del 5\%, la información aportada por las variables explicativas es significativamente mejor que la que podría aportar únicamente la media.

Tabla 3. Análisis de varianza $(\mathrm{pH})$

\begin{tabular}{|c|c|c|c|c|c|}
\hline Fuente & GL & $\begin{array}{l}\text { Suma de } \\
\text { cuadrados }\end{array}$ & Cuadrados medios & $\mathrm{F}$ & $\operatorname{Pr}>F$ \\
\hline Modelo & 1 & 0.727 & 0.727 & 55.639 & $<0.0001$ \\
\hline Error & 11 & 0.144 & 0.013 & & \\
\hline $\begin{array}{l}\text { Total } \\
\text { corregido }\end{array}$ & 12 & 0.870 & & & \\
\hline
\end{tabular}


Del análisis de regresión lineal para el parámetro EC se obtiene los estadísticos de bondad de ajuste (tabla 4), el mismo que da un índice de determinación de $\mathrm{R}^{2}=0.782$, que indica que el EC es dependiente del Tiempo de exposición en minutos en un 78.2 \% y se debe a otros factores en un $21.8 \%$.

Tabla 4. Estadísticos de bondad de ajuste para EC

\begin{tabular}{ll}
\hline Observaciones & 13.000 \\
Suma de los pesos & 13.000 \\
$\mathrm{GL}$ & 11.000 \\
$\mathrm{R}^{2}$ & 0.782 \\
$\mathrm{R}^{2}$ ajustado & 0.762 \\
\hline
\end{tabular}

La figura 10 muestra la regresión lineal del EC con una línea de tendencia con pendiente negativa (-0.68), lo que indica que aun podrá disminuir la cantidad de sales en el agua a medida que se incremente el tiempo de exposición.

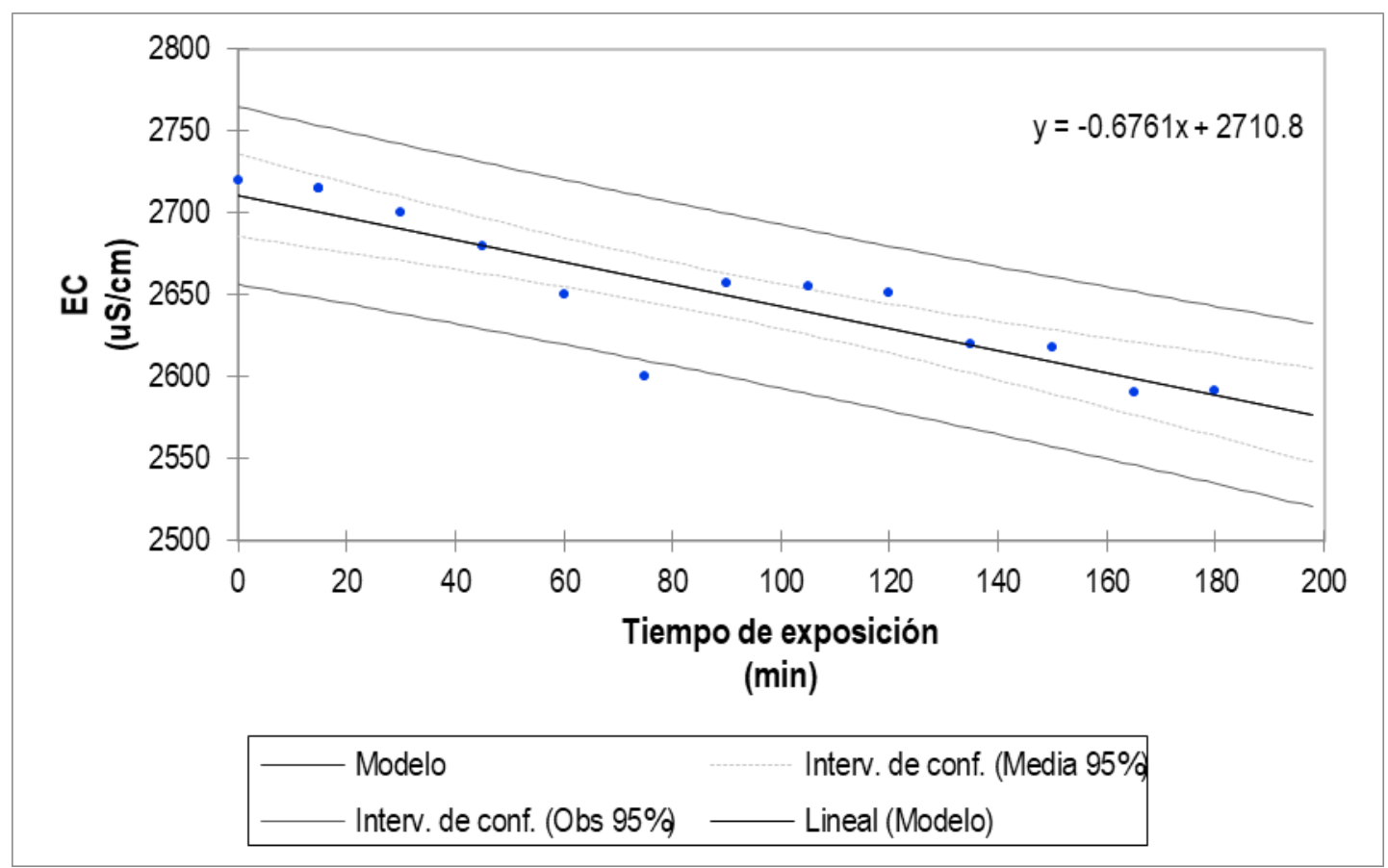

Figura 10. Regresión lineal de EC por Tiempo de exposición

En la tabla 5 se presenta el análisis de varianza para el EC; dado el valor p (< 0.0001) asociado al estadístico F calculado (39.423) en la tabla ANOVA, y dado el 

nivel de significación del 5\%, la información aportada por las variables explicativas es significativamente mejor que la que podría aportar únicamente la media.

Tabla 5. Análisis de varianza (EC)

\begin{tabular}{|c|c|c|c|c|c|}
\hline Fuente & GL & $\begin{array}{l}\text { Suma de } \\
\text { cuadrados }\end{array}$ & $\begin{array}{l}\text { Cuadrados } \\
\text { medios }\end{array}$ & $\mathrm{F}$ & $\operatorname{Pr}>F$ \\
\hline Modelo & 1 & 18717.629 & 18717.629 & 39.423 & $<0.0001$ \\
\hline Error & 11 & 5222.742 & 474.795 & & \\
\hline $\begin{array}{l}\text { Total } \\
\text { corregido }\end{array}$ & 12 & 23940.371 & & & \\
\hline
\end{tabular}

Del análisis de regresión lineal para el parámetro DO se obtiene los estadísticos de bondad de ajuste (tabla 6 ), el mismo que da un índice de determinación de $\mathrm{R}^{2}=0.863$, que indica que el DO es dependiente del Tiempo de exposición en minutos en un 86.3 $\%$ y se debe a otros factores en un $13.7 \%$.

Tabla 6. Estadísticos de bondad de ajuste para DO

\begin{tabular}{ll}
\hline Observaciones & 13.000 \\
Suma de los pesos & 13.000 \\
GL & 11.000 \\
$\mathrm{R}^{2}$ & 0.863 \\
$\mathrm{R}^{2}$ ajustado & 0.851 \\
\hline
\end{tabular}

La figura 11 muestra la regresión lineal del DO con una línea de tendencia con pendiente positiva (0.006), lo que indica que aun podrá incrementar el oxígeno disuelto en el agua a medida que se incremente el tiempo de exposición. 


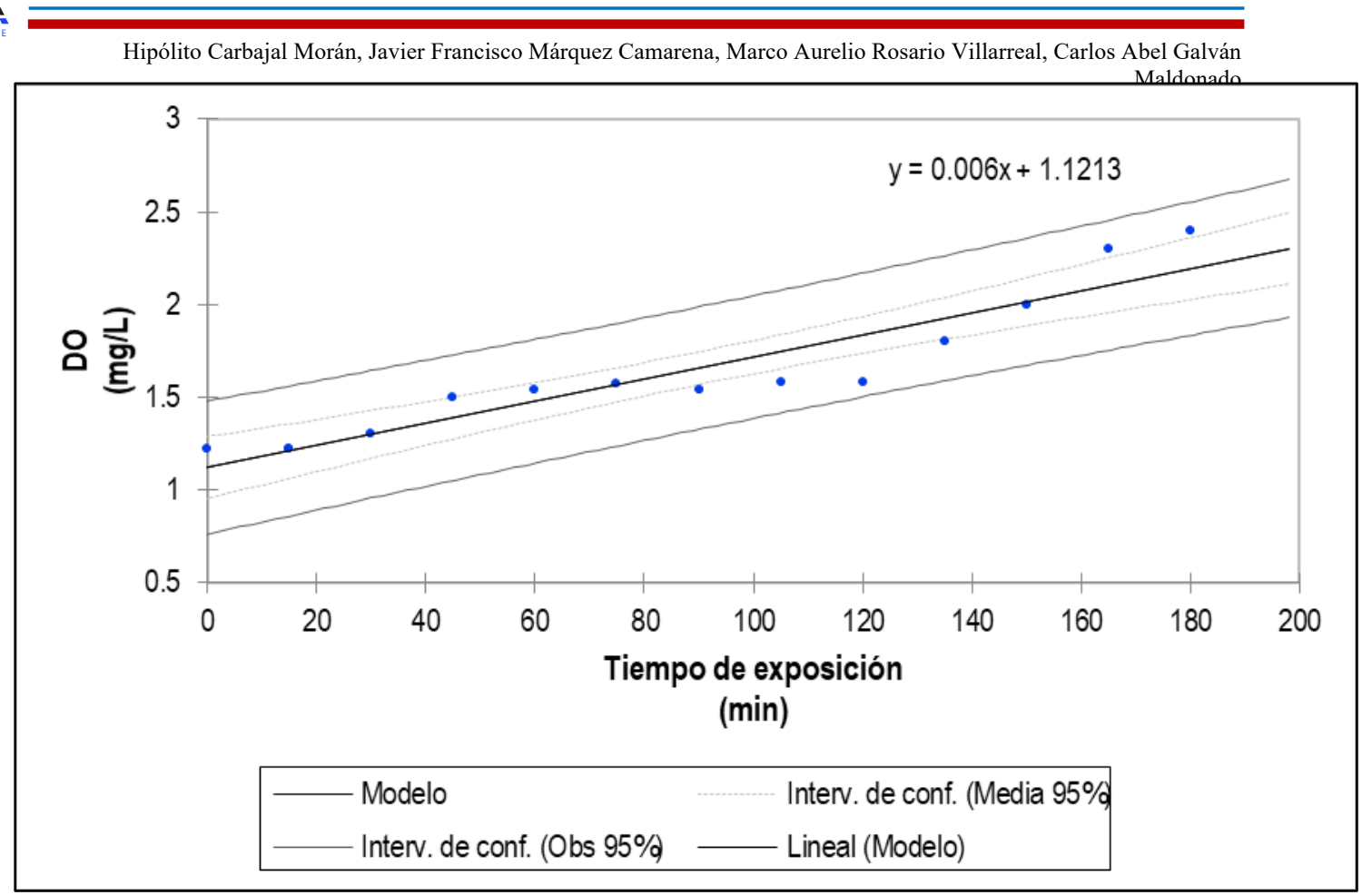

Figura 11. Regresión lineal de DO por Tiempo de exposición

En la tabla 7 se presenta el análisis de varianza para el DO; dado el valor p (< 0.0001) asociado al estadístico F calculado (39.423) en la tabla ANOVA, y dado el nivel de significación del 5\%, la información aportada por las variables explicativas es significativamente mejor que la que podría aportar únicamente la media.

Tabla 7. Análisis de varianza (DO)

\begin{tabular}{|c|c|c|c|c|c|}
\hline Fuente & GL & $\begin{array}{l}\text { Suma de } \\
\text { cuadrados }\end{array}$ & $\begin{array}{l}\text { Cuadrados } \\
\text { medios }\end{array}$ & $\mathrm{F}$ & $\operatorname{Pr}>F$ \\
\hline Modelo & 1 & 18717.629 & 18717.629 & 39.423 & $<0.0001$ \\
\hline Error & 11 & 5222.742 & 474.795 & & \\
\hline $\begin{array}{l}\text { Total } \\
\text { corregido }\end{array}$ & 12 & 23940.371 & & & \\
\hline
\end{tabular}

Del análisis de regresión lineal para el parámetro FCL se obtiene los estadísticos de bondad de ajuste (tabla 8 ), el mismo que da un índice de determinación de $\mathrm{R}^{2}=$ 0.941, que indica que el FCL es dependiente del Tiempo de exposición en minutos en un $94.1 \%$ y se debe a otros factores en un $5.9 \%$. 
Tabla 8. $\quad$ Estadísticos de bondad de ajuste para FCL

\begin{tabular}{ll}
\hline Observaciones & 13.000 \\
Suma de los pesos & 13.000 \\
$\mathrm{GL}$ & 11.000 \\
$\mathrm{R}^{2}$ & 0.941 \\
$\mathrm{R}^{2}$ ajustado & 0.936 \\
\hline
\end{tabular}

La figura 12 muestra la regresión lineal del FCL con una línea de tendencia con pendiente negativa (-0.0121), lo que indica que aun podrá disminuir la concentración de cloro libre en el agua a medida que se incremente el tiempo de exposición.

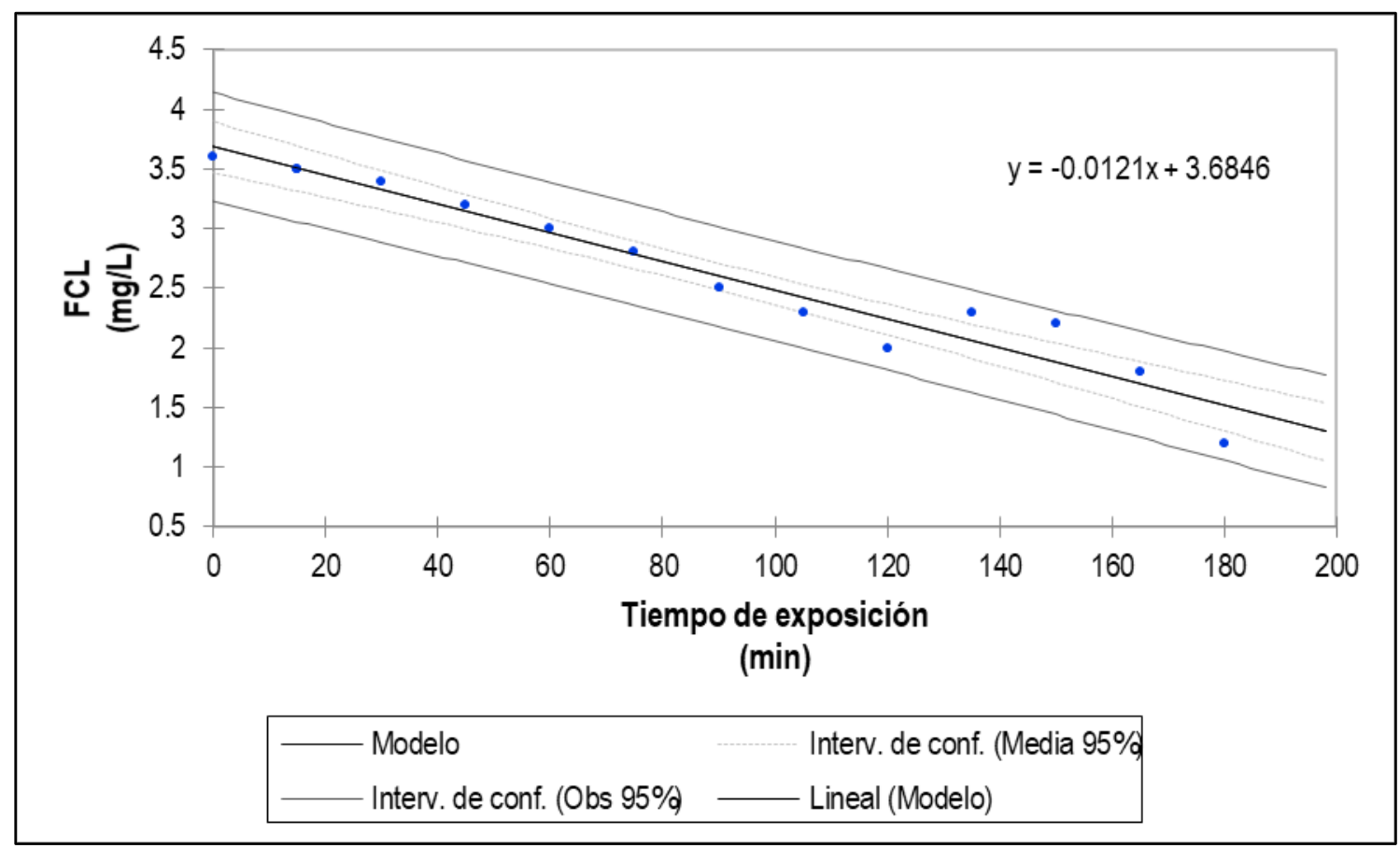

Figura 12. Regresión lineal de FCL por Tiempo de exposición

En la tabla 9 se presenta el análisis de varianza para el FCL; dado el valor p $(<$ 0.0001) asociado al estadístico F calculado (175.54) en la tabla ANOVA, y dado el nivel de significación del 5\%, la información aportada por las variables explicativas es significativamente mejor que la que podría aportar únicamente la media. 
Tabla 9. $\quad$ Análisis de varianza (FCL)

\begin{tabular}{|c|c|c|c|c|c|}
\hline Fuente & GL & $\begin{array}{l}\text { Suma de } \\
\text { cuadrados }\end{array}$ & $\begin{array}{l}\text { Cuadrados } \\
\text { medios }\end{array}$ & $\mathrm{F}$ & $\operatorname{Pr}>F$ \\
\hline Modelo & 1 & 5.947 & 5.947 & 175.535 & $<0.0001$ \\
\hline Error & 11 & 0.373 & 0.034 & & \\
\hline $\begin{array}{l}\text { Total } \\
\text { corregido }\end{array}$ & 12 & 6.320 & & & \\
\hline
\end{tabular}

\section{Discusión}

Se aplicó el análisis de regresión lineal para el análisis de los parámetros $\mathrm{pH}, \mathrm{EC}$, DO y FCL en función del tiempo de exposición a la radiación solar UV, obteniendo variaciones significativas de los parámetros en estudio mediante el ANOVA para cada parámetro (tablas 3, 5, 7 y 9), aproximándose a los valores establecidos por el ministerio del ambiente (MINAM, 2017a), siendo coherente con el trabajo de Rincón y Pulgarin (2007) y el trabajo de Nahim et al (2018), donde analizaron aguas contaminadas en la industria con alto nivel de turbidez demostrando con procesos manuales con $\mathrm{H}_{2} \mathrm{O} /$ solar y la influencia de la irradiación UVA $\left(10-50 \mathrm{~W} / \mathrm{m}^{2}\right)$ contra la efectiva depuración de agua, siendo una limitante los procesos repetitivos. Este aspecto fue superado por esta investigación ya que se emplea un sistema electrónico programable basado en PLC S7 1500, módulo de adquisición de datos y sensores online.

Otro estudio es el presentado por Almomani et al. (2018), donde se utilizó una planta piloto para evaluar el potencial de los procesos fotocatalíticos para eliminar contaminantes emergentes, por productos farmacéuticos, los experimentos de oxidación se realizaron, bajo luz solar natural y a temperatura ambiente, en un fotorreactor. Los resultados muestran que se requiere de elementos fotoactivos para eliminar productos farmacéuticos de las aguas residuales, llegando a una eficacia del 90\%. Por lo que es necesario mejorar en futuras investigaciones usar fotorreactores para mejorar el proceso de recuperación de aguas grises.

Del mismo modo Barwal y Chaudhary (2016) desarrollaron un reactor acoplado a un sistema parabólico para remover la carga orgánica en agua que es sometido a la energía solar directamente con tiempos extensos de 5 a 6 horas, en nuestra investigación el experimento se llevó a cabo durante 3 horas, donde algunos factores se aproximaron a los valores deseados como es el caso del $\mathrm{pH}$. 


\section{Bibliografía}

Akiça, V. L. (2004). Water Reuse for Water Reuse for Irrigation, Agriculture, Landscapes, and Turf Grass. En New York. CRC PRESS. https://books.google.com.pe/books?id=cG2ueBvLc0oC\&printsec=copyright

Almomani, F., Bhosale, R., Kumar, A., \& Khraisheh, M. (2018). Potential use of solar photocatalytic oxidation in removing emerging pharmaceuticals from wastewater: A pilot plant study. Solar Energy. https://doi.org/10.1016/J.SOLENER.2018.07.041

Barwal, A., \& Chaudhary, R. (2016). Feasibility study for the treatment of municipal wastewater by using a hybrid bio-solar process. Journal of Environmental Management, 177, 271-277. https://doi.org/10.1016/J.JENVMAN.2016.04.022

BOPLA. (2019). Grado de protección IP 68. https://www.bopla.de/es/datostecnicos/informaciones-tecnicas/grados-de-proteccion/ip-68.html

Boyd, C. E. (2020). Water quality (U. Auburn (ed.); 3. a ed.). Springer. https://doi.org/10.1007/978-3-030-23335-8

Cegarra Sánchez, J. (2012). Metodología de la investigaión científica y Tecnológica. Books Médicos. https://books.google.es/books?hl=es\&lr=\&id=YROO_q6wzgC\&oi=fnd\&pg=PA81\&dq=método + hipotético + deductivo + metodología\&ots $=$ Y22eZFpaNR\&sig=oUTM8Xxoa20LMC1KwIcO8zFsB_0\#v=onepage\&q=métod o hipotético deductivo metodología\&f=fals

EMRC. (2011). Reuse of Greywater in Western Australia. Discussion Paper Australia.

FAO. (2017). Reutilización de aguas para agricultura en América Latina y el Caribe: Estado, principios y necesidades. http://www.fao.org/3/a-i7748s.pdf

Franco, M. V. (2007). Tratamiento y reutilización de aguas grises con aplicación a caso en Chile. 1-142. http://repositorio.uchile.cl/handle/2250/104596

Larios, J. F., Gonzales, C., \& Morales, Y. (2015). Las aguas residuales y sus consecuencias en el Perú. Revista de la Facultad de Ingeniería de la USIL. https://doi.org/https://doi.org/10.1016/S0011-9164(96)00124-5

Mehta, L. (2014). Water and human development. World Development, 59, 59-69. https://doi.org/10.1016/j.worlddev.2013.12.018

MINAM. (2017a). Decreto Supremo Nº04-2017-MINAM. https://sinia.minam.gob.pe/normas/aprueban-estandares-calidad-ambiental-ecaagua-establecen-disposiciones 
MINAM. (2017b). Estándares de Calidad Ambiental (ECA) para Agua. El Peruano, 19. https://www.minam.gob.pe/wp-content/uploads/2017/06/DS-004-2017-

MINAM.pdf

Nahim, S., Sánchez, J. A., \& Polo, M. I. (2018). Effective solar processes in fresh-cut wastewater disinfection: Inactivation of pathogenic E. coli O157:H7 and Salmonella enteritidis. En Catalysis Today (4, Vol. 313). Elsevier. https://doi.org/10.1016/J.CATTOD.2017.10.042

Niño, E. D., \& Martínez, N. C. (2014). Estudio de las aguas grises domésticas en tres niveles socioeconómicos de la ciudad de Bogotá [B.S. thesis].

Peciña, L. (2018). Programación de Controladores avanzados SIMATIC S7 1500 con TIA Portal AWL y SCL (2. ${ }^{a}$ ed.). Marcombo.

REMOND. (2019). Water quality analysis. https://remond.en.alibaba.com/es_ES/?spm=a2700.icbuShop.88.7.20674367QPvk OI

Rincón, A. G., \& Pulgarin, C. (2007). Solar photolytic and photocatalytic desinfection of water at laboratory and field scale. Effect of the chemical composition of water and study of the post irradiation events. 23 .

SENAMHI. (2019). Pronóstico de radiación UV máximo (cielo despejado y mediodía solar) a nivel nacional. https://www.senamhi.gob.pe/?\&p=radiacion-uv

Siemen. (2018). S7-1500/ET 200MP Módulo de entradas analógicas AI 8xU/I/RTD/TC ST (6ES7531-7KF00-0AB0).

https://cache.industry.siemens.com/dl/files/205/59193205/att_112068/v1/s71500_ ai_8xu_i_rtd_tc_st_manual_es-ES_es-ES.pdf

Siemens. (2013). S7-1500 Sistema de automatización. 1-214.

https://support.industry.siemens.com/cs/attachments/59191792/s71500_et200mp_ system_manual_es-ES_es-ES.pdf?download=true

Siemens. (2017). TIA Portal V15.

https://rive.google.com/file/d/1W4nYR0u93OMcOexz44OpnWtGQedwg7Ve/vi ew

Silva, J., Torres, P., \& Madera, C. (2008). Reuso de aguas residuales domésticas en agricultura. Una revisión. Agronomía Colombiana, 26(2).

Valencia, E., Romero, J., \& Aragón, R. A. (2010). Esquema metodológico para la reutilización de aguas residuales domésticas tratadas en riego. 2010 (Enero- 
Diciembre);(9): p. 55-59. Ingeniería de Recursos Naturales y del Ambiente, 9 , 5 , 5 59.

Ventura, V. (2016). Sensor de radiación ultravioleta con Arduino y UVM30A.

https://polaridad.es/sensor-radiacion-ultravioleta-arduino-indice-uv-uvm30aguva-s12sd/ 